\title{
Efficient Hybrid Optimal Design Method for Power Electronics Converters
}

Francisco Cabaleiro Magallanes, Davide Aguglia

CERN, Geneva, Switzerland, Philippe Viarouge, Jérôme Cros

LEEPCI Lab, Québec, Canada

Keywords: «Pulsed Power», «Pulsed power converter», «Power supply», «Design», «Converter circuit».

\begin{abstract}
This paper presents a novel design methodology for dimensioning optimal power-electronic converters, which is able to achieve the precision of numerical simulation-based optimization procedures, however minimizing the overall computation time. The approach is based on the utilization of analytical and frequency-domain design models for a numerical optimization process, a validation with numerical simulations of the intermediate optimal solutions, and the correction of the analytical design models precision from the numerical simulation results. This method allows using the numerical simulation in an efficient way, where typically less than ten correction iterations are required. In order to demonstrate the performances of the proposed methodology, the calculation of the control parameters for an H-bridge DC-DC converter and the optimal dimensioning of a damped output filter for a buck converter using the proposed approach is presented.
\end{abstract}

Presented at: EPE 2015, 7-10 September 2015, Geneva, Switzerland

Geneva, Switzerland

October, 2015 


\title{
Efficient Hybrid Optimal Design Method for Power Electronics Converters
}

\author{
Francisco Cabaleiro Magallanes ${ }^{1,2}$, Davide Aguglia ${ }^{1}$, Philippe Viarouge ${ }^{2}$, and Jérôme Cros ${ }^{2}$ \\ ${ }^{1}$ CERN-EUROPEAN ORGANIZATION FOR NUCLEAR RESEARCH \\ 1217 Geneva 23, Switzerland \\ Tel.: +41 / (0) - 227678489 . \\ E-Mails: fcabalei@cern.ch,davide.aguglia@cern.ch \\ URL: http://www.cern.ch \\ ${ }^{2}$ LEEPCI Lab., Laval University, Electrical and Computer Eng. Dept. \\ GIK 7P4, Québec (QC), Canada \\ Tel.: +1/ 41865621317139 \\ E-Mail: philippe.viarouge@ulaval.ca \\ URL: http://leepci.gel.ulaval.ca
}

\section{Keywords}

«Pulsed Power», «Pulsed power converter», «Power supply», «Design», «Converter circuit».

\begin{abstract}
This paper presents a novel design methodology for dimensioning optimal power-electronic converters, which is able to achieve the precision of numerical simulation-based optimization procedures, however minimizing the overall computation time. The approach is based on the utilization of analytical and frequency-domain design models for a numerical optimization process, a validation with numerical simulations of the intermediate optimal solutions, and the correction of the analytical design models precision from the numerical simulation results. This method allows using the numerical simulation in an efficient way, where typically less than ten correction iterations are required. In order to demonstrate the performances of the proposed methodology, the calculation of the control parameters for an H-bridge DC-DC converter and the optimal dimensioning of a damped output filter for a buck converter using the proposed approach is presented.
\end{abstract}

\section{Introduction}

Important efforts have been made during the last 30 years to optimize power converter designs. Several solutions in the literature proposed different methods to maximize the robustness and the efficiency, or minimize weight, volume and cost, by modifying whether the control parameters or directly the power converter design. A simultaneous optimization considering control and design parameters as optimization variables has also been presented in [1]. Genetic Algorithms (GAs) [2][3][4], Augmented Lagrangian (ALAG) [5][6][7], and Sequential quadratic programming (SQP) techniques [8], are usually the selected optimization procedures.

In order to speed-up the computation time, the design models are usually based on analytical formulation. Moreover, the designer also introduces simplifications and design constraints from previous design experience. As a consequence, their accuracy is reduced in comparison with simulation-based optimization techniques, which make possible to easily consider delays, saturations, and other nonlinearities present in the real system. However, the important computation time required for performing a numerical simulation on every optimization step/iteration, usually forces the designer to reduce the optimization variables to a reduced subset. In this paper we apply to power electronics systems a hybrid optimal design methodology which has been successfully proposed and used to efficiently optimize electrical machines considering magnetic saturation effects [9]. 


\section{Design Method Description}

As an alternative to achieve a trade-off between design time and accuracy, this paper presents a hybrid optimization procedure whose scheme is illustrated in Figure 1. The proposed strategy starts by selecting a power converter topology, by setting the optimization objectives in terms of minimum performances that must be respected (constraints), the performances or parameters that must be optimized (e.g. bandwidth, efficiency, etc.), and by selecting the optimization variables (controller parameters only or/and power stage component values). Then, after an initialization of the optimization variables, which are set by the designer expertise, a first optimization routine starts, where the performances are evaluated via frequency-domain analysis of the closed loop transfer function (classical design method based on Bode plots). Once an optimal solution is obtained, a timedomain simulation with the calculated parameters is carried out. If differences are found between the analytically predicted performances and the ones obtained in the numerical simulation, the frequencydomain models are modified/corrected via correction factors, and the optimization routine is restarted again. It will be shown that, after a limited number of corrective iterations, the analytical and numerical models converge. At the end of the whole procedure illustrated in Figure 1, the final optimal solution is naturally numerically validated. It is interesting to note that during the optimization procedure the corrective factors for the analytical model can be saved at each corrective iteration. This allows the designer to appreciate the exactness of the initial analytical model and the evolution in its correction. This method offers a good compromise between the speed of the analytical model-based optimization approach and, at the same time, it provides the accuracy of the time-domain numerical simulation-based models.

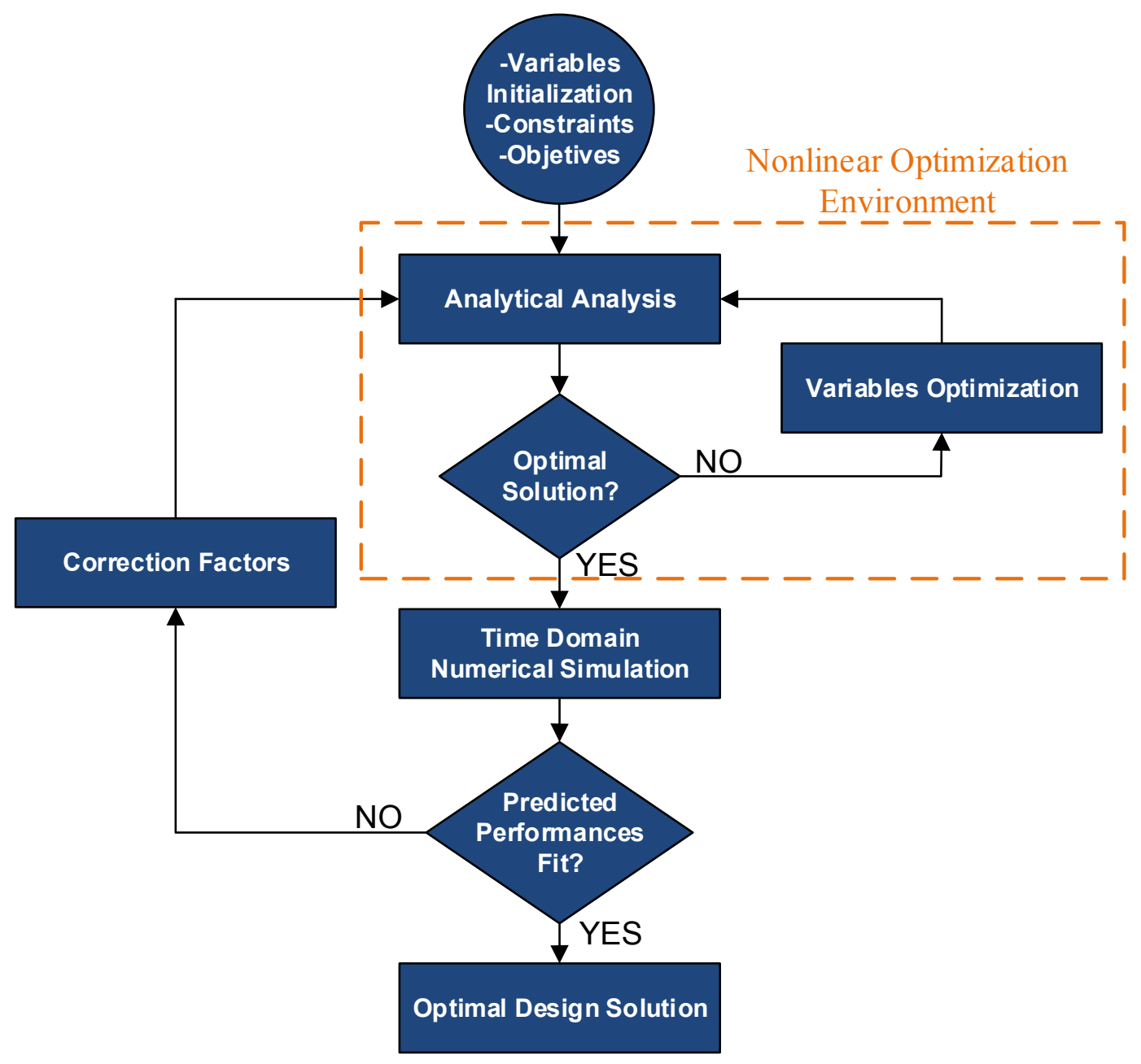

Fig. 1: Proposed optimization scheme integrating analytical and numerical models. 
To illustrate the power of such an optimization procedure, two design examples are considered. The first one includes typical nonlinearities which would normally lead to complex analytical design formulations. The second example illustrates the optimal design of a converter power parts respecting its initially defined functional specifications and integrating the maximization of its efficiency.

\section{Example 1: Controller optimization of a non-linear power converter}

\section{Design problem definition}

In order to illustrate the capabilities of the proposed design method, a first optimization of the controller parameters (PI) of an H-bridge 4-quadrant DC-DC converter, with the parameters summarized in Table 1, is presented. The optimization objective consists in maximizing the closedloop bandwidth of the system $\omega_{\mathrm{BW}}$, while respecting a maximum closed-loop step response overshoot of $27 \%$ and a minimum phase margin of $30^{\circ}$.

Table 1 Power converter fixed parameters

\begin{tabular}{|l|c|c|}
\hline \multicolumn{1}{|c|}{ PARAMETER } & SYMBOL & VALUE \\
\hline DC Bus Voltage & $\mathrm{V}_{\mathrm{DC} \text { BUS }}$ & $1000 \mathrm{~V}$ \\
\hline Filter Inductance & $\mathrm{L}$ & $50 \mu \mathrm{H}$ \\
\hline Filter Capacitance & $\mathrm{C}$ & $22 \mu \mathrm{F}$ \\
\hline Resistive Load & $\mathrm{R}_{\text {load }}$ & $0.5 \Omega$ \\
\hline
\end{tabular}

\section{Analytical model}

A simplified analytical model is used to evaluate the closed-loop system transfer function as shown in (1), which includes the PI regulator parameters $K_{p}$ and $T_{i}$ (proportional and integral coefficients, respectively) and the system parameters of Table 1 . The closed-loop bandwidth $\omega_{\mathrm{BW}}$ of the system is calculated by solving equation (2). The crossover frequency for the gain $\omega_{\mathrm{GC}}$ is calculated as shown in (3). With this value the phase margin can be calculated as shown in (4). Finally, simplifying to the first harmonic, the overshoot constraint can be expressed in the frequency domain as in (5), where the factor 1.27 relates to the specified $27 \%$ overshoot in the time domain.

$$
\begin{aligned}
& G_{C L}(s)=\frac{K_{P} \cdot R \cdot T_{i} \cdot S+K_{P} \cdot R}{C \cdot L \cdot R \cdot T_{i} \cdot S^{3}+L \cdot T_{i} \cdot S^{2}+\left(R \cdot T_{i}+K_{P} \cdot R \cdot T_{i}\right) s+K_{P} \cdot R} \\
& -3 d B=20 . \log _{10}\left(\frac{\sqrt{\left(K_{P} \cdot R \cdot T_{i} \cdot \omega_{B W}\right)^{2}+\left(K_{P} \cdot R\right)^{2}}}{\sqrt{\left(\left(R \cdot T_{i}+K_{P} \cdot R \cdot T_{i}\right) \cdot \omega_{B W}-C \cdot L \cdot R \cdot T_{i} \cdot \omega_{B W}^{3}\right)^{2}+\left(K_{P} \cdot R-L \cdot T_{i} \cdot \omega_{B W}^{2}\right)^{2}}}\right) \\
& 0 d B=20 . \log _{10}\left(\frac{\sqrt{\left(K_{P} \cdot R \cdot T_{i} \cdot \omega_{G C}\right)^{2}+\left(K_{P} \cdot R\right)^{2}}}{\sqrt{\left(\left(R \cdot T_{i}+K_{P} \cdot R \cdot T_{i}\right) \cdot \omega_{G C}-C \cdot L \cdot R \cdot T_{i} \cdot \omega_{G C}^{3}\right)^{2}+\left(K_{P} \cdot R-L \cdot T_{i} \cdot \omega_{G C}^{2}\right)^{2}}}\right) \\
& P M=\pi+\tan ^{-1}\left(\frac{K_{P} \cdot R \cdot T_{i} \cdot \omega_{G C}}{K_{P} \cdot R}\right)-\tan ^{-1}\left(\frac{\left(R \cdot T_{i}+K_{P} \cdot R \cdot T_{i}\right) \cdot \omega_{G C}-C \cdot L \cdot R \cdot T_{i} \cdot \omega_{G C}^{3}}{K_{P} \cdot R-L \cdot T_{i} \cdot \omega_{G C}^{2}}\right) \\
& 20 . \log _{10}(1.27)>k_{1} \cdot \max \left(20 \cdot \log _{10}\left(\frac{\sqrt{\left(K_{P} \cdot R \cdot T_{i} \cdot \omega\right)^{2}+\left(K_{P} \cdot R\right)^{2}}}{\sqrt{\left(\left(R \cdot T_{i}+K_{P} \cdot R \cdot T_{i}\right) \cdot \omega-C \cdot L \cdot R \cdot T_{i} \cdot \omega^{3}\right)^{2}+\left(K_{P} \cdot R-L \cdot T_{i} \cdot \omega^{2}\right)^{2}}}\right)\right)
\end{aligned}
$$

As this analytical formulation is based on many hypothesis (first harmonic approximation, no saturation effects, etc.), one can foresee to correct one or more than one of the above analytical equations with the proposed design method. For illustration and simplicity purposes, it has been decided to foresee the correction of the overshoot estimation in (5). Indeed, a correction factor $k_{1}$, is 
added to this expression to increase the accuracy of the analytical model. Its derivation will be presented hereafter.

The non-linear constrained optimization process takes place and is based on the above mentioned analytical model. At the end of this process an optimal design solution, based on the inaccurate analytical model, is obtained. This intermediate optimal design solution is afterwards numerically simulated in the time domain.

\section{Time domain numerical simulation and corrective factor derivation}

Figure 2 illustrates the considered model for time domain numerical simulation. This model takes into account phenomena which were not considered in the analytical model, used for optimization purposes. In this model all harmonics are naturally considered and a saturation, representing the DCBus voltage limitation of this converter, has been integrated.

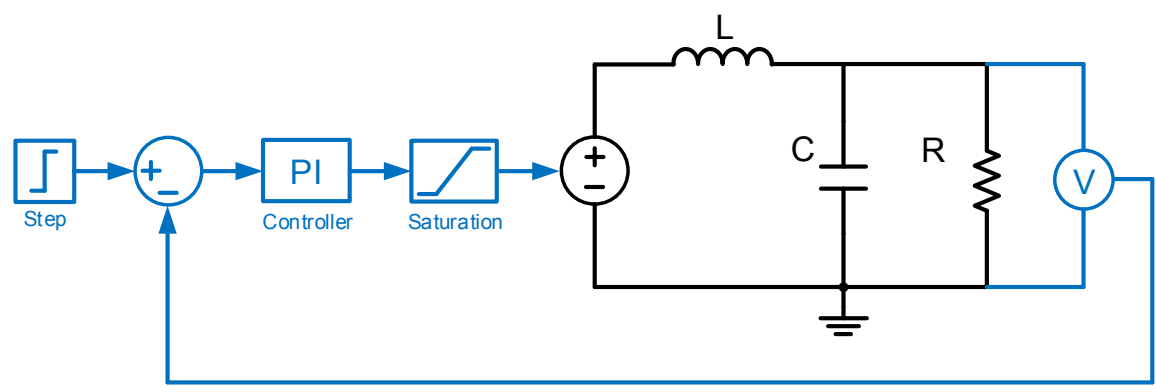

Fig. 2: H-bridge DC-DC converter numerical simulation model.

From the numerical simulation one derives the actual voltage overshoot peak (Ovs) obtained from a step response. At this point the measured overshoot can be translated into an equivalent Gain in the frequency domain (in $\mathrm{dB}$ ), still with first harmonic hypothesis, as shown in (6).

$$
\text { Overshoot }(d B)=20 \cdot \log _{10}\left(\frac{\text { ovs }}{V_{D C_{-} B U S}}\right)
$$

Then, the correction factor $k_{l}$ can be derived as in (7).

$$
k_{1}=\frac{\text { overshoot }(\mathrm{dB})}{\text { Peak predicted by analytical design models (right hand side of }(5))}
$$

At this point the analytical model is corrected, and the process can restart with a new optimization procedure based on the newly corrected model (via $\left.k_{l}\right)$.

\section{Optimal design process summary for Example 1}

The sequential hybrid optimization procedure can be summarized as follows:

1. Set initial conditions for the PI controller $\left(K_{p}\right.$ and $\left.T_{i}\right)$ and $k_{l}=1$.

2. Start non-linear constrained optimization process based on the analytical method.

a. Compute the bandwidth $\boldsymbol{\omega}_{\mathrm{BW}}$ from (2).

b. Compute phase margin PM from (3) and (4).

c. Compute the overshoot from right hand side of (5).

d. Check optimality (a.) and constraints (b. \& c.) and iteration on $K_{p}$ and $T_{i}$ (variables).

e. Restart optimization iterations until reaching optimality and respecting constraints.

3. Transfer optimal parameters to time domain numerical simulation and start simulation. 
4. From simulation results compute the correction factor $k_{l}$

5. Restart from point 2. until the error between analytical and numerical results are within a predefined limit. .

The passage from point 4 . to point 5 . is called "corrective iteration step".

\section{Example 1 design optimization results}

Figure 3 shows the evolution of the overshoot peak predicted by the analytical design models and the one achieved in the numerical simulation, for each corrective iteration step. Figure 4 shows how, after 9 corrective iteration steps, the absolute error of the predicted overshoot peak during the step response drops below 10\%; and the final correction factor approaches 7 .

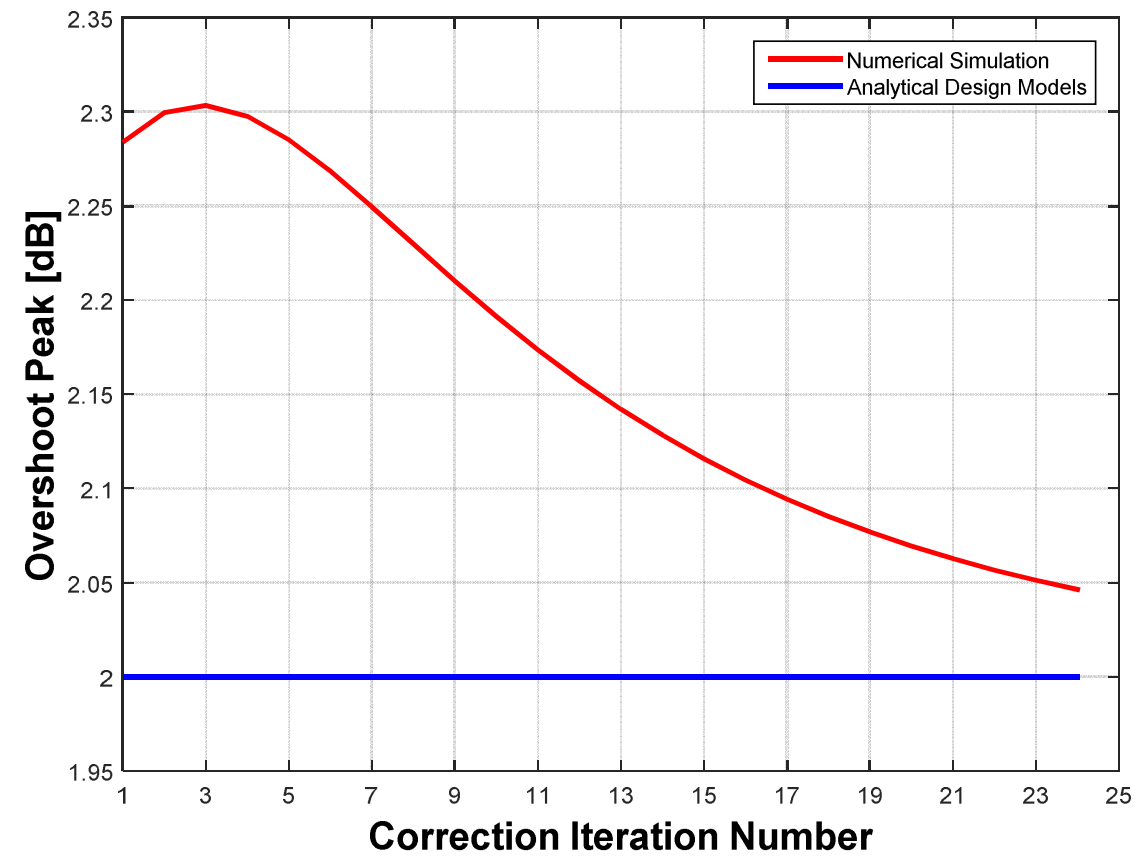

Fig. 3: Evolution of the overshoot peak predicted by the frequency domain design models versus the ones obtained by numerical simulation
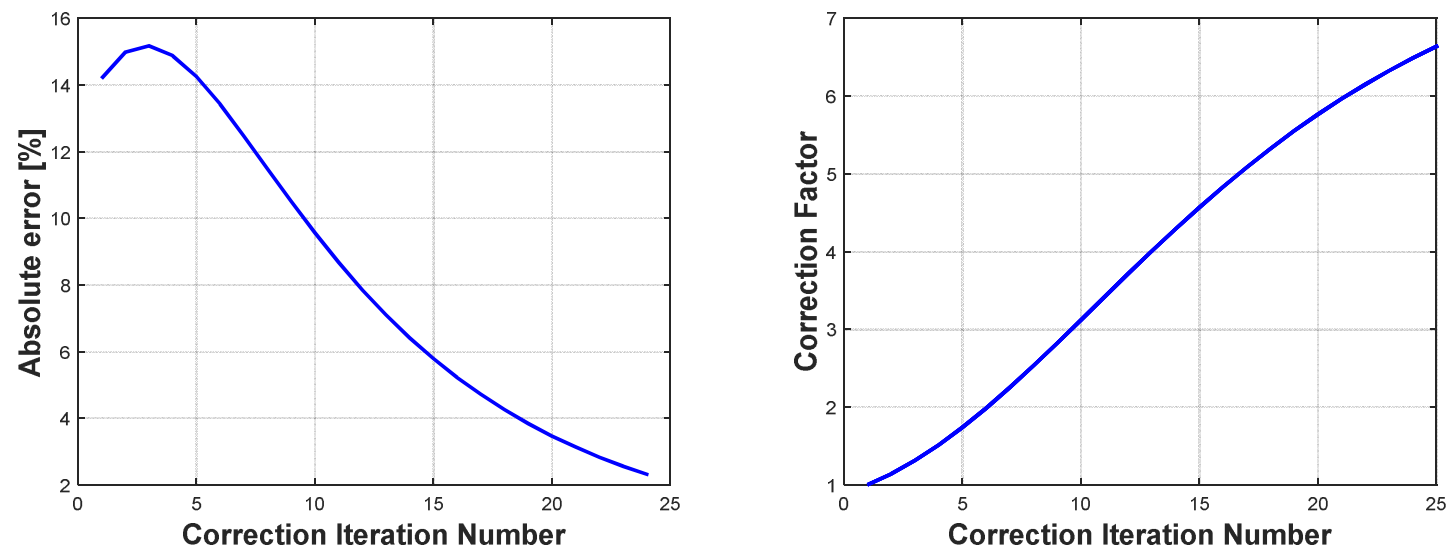

Fig. 4: Evolution of the absolute error on overshoot and correction factor during 24 iteration steps.

The final correction factor value is relatively high. Indeed, this means that the analytical formulation of the overshoot is highly inaccurate for this specific optimization procedure. This is due to the nonlinearity effect of the DC-Bus voltage limitation. This saturation is not being considered into the 
analytical method and, in this case where the bandwidth has to be maximized, it is clear that the output of the PI controller is saturated during the vast majority of the voltage step-up time. Even with a highly inaccurate analytical formulation, the method could converge to an optimal, and at the same time numerically validated, solution in 25 corrective iteration steps only.

\section{Example 2: Optimization of a Damped Output Filter}

In this second example we illustrate the application of the proposed method to the dimensioning of a buck converter equipped with a passively damped output filter and a resistive load, as illustrated in Figure 5. The power converter must respect the specifications shown in Table 2.

Table 2 Specifications

\begin{tabular}{|l|c|c|}
\hline \multicolumn{1}{|c|}{ PARAMETER } & SYMBOL & VALUE \\
\hline DC Bus Voltage & $\mathrm{V}_{\mathrm{DC} \text { BUS }}$ & $1000 \mathrm{~V}$ \\
\hline Resistive Load & $\mathrm{R}$ & $1 \mathrm{k} \Omega$ \\
\hline Desired Output Voltage & $\mathrm{V}_{\text {OUT }}$ & $500 \mathrm{~V}$ \\
\hline Maximum Rise Time & $\mathrm{T}_{\text {RISE MAX }}$ & $200 \mu \mathrm{s}$ \\
\hline Minimum attenuation of switching frequency harmonics & $\mathrm{A}_{\mathrm{FS}}$ & $40 \mathrm{~dB}$ \\
\hline Maximum overshoot peak & $\mathrm{A}_{\mathrm{OV}}$ & $1 \mathrm{~dB}$ \\
\hline Flat-Top length & $\mathrm{T}_{\mathrm{PULSE}}$ & $2 \mathrm{~ms}$ \\
\hline Repetition rate & $\mathrm{RR}$ & $20 \mathrm{~Hz}$ \\
\hline
\end{tabular}

The power converter has to produce $2 \mathrm{~ms}$ pulses of $500 \mathrm{~V}$ with a repetition rate of $20 \mathrm{~Hz}$. The objective function consists in minimizing the sum of the converter capital cost and the operating cost due to the total losses (switching and conduction losses in IGBT $T 1$, conduction and reverse recovery losses in free-wheeling diode $D 1$, losses on damping resistor $R_{D}$ and losses on inductance $L$ ) over a period of 5 years. Output capacitors ESR will be neglected. The optimization variables are: the switching frequency, $L, C, R d$ and $C d$ values. After each analytical optimization step, the overshoot peak and the ripple attenuation values are verified by numerical simulation and correction factors applied to the analytical models to improve their precision. In this case the open-loop transfer function of the system is:

$$
G_{C L}(s)=\frac{C_{d} \cdot R_{d} \cdot R \cdot s+R}{C \cdot C_{d} \cdot R_{d} L \cdot R \cdot s^{3}+\left(C \cdot L \cdot R+C_{d} \cdot L \cdot R+C_{d} \cdot L \cdot R_{d}\right) \cdot s^{2}+\left(C_{d} \cdot R_{d} \cdot R+L\right) \cdot s+R}
$$

The overshoot (in dBs) of the system can be calculated in the same way as in Example 1, including the same correction factor $k_{l}$.

$k_{1} \cdot \max \left(20 \cdot \log _{10}\left(\frac{\sqrt{\left(C_{d} \cdot R_{d} \cdot R \cdot \omega_{f s}\right)^{2}+(R)^{2}}}{\sqrt{\left(\left(C_{d} \cdot R_{d} \cdot R+L\right) \cdot \omega_{f s}-C \cdot C_{d} \cdot R_{d} L \cdot R \cdot \omega_{f s}^{3}\right)^{2}+\left(R-\left(C \cdot L \cdot R+C_{d} \cdot L \cdot R+C_{d} \cdot L \cdot R_{d}\right) \cdot \omega_{f s}^{2}\right)^{2}}}\right)\right)$

The minimum bandwidth of the system is computed using the classical relation between bandwidth of a signal and its rise time (to be respected from the specification in Table 2) as shown in (10). The attenuation of the switching frequency harmonics is calculated as shown in (11). A correction factor $k_{2}$ has been added in order to correct this expression. Again, only the first harmonic of the input voltage waveform has been taken into consideration in the analytical formulation as a simplification.

$$
R T(s)=\frac{0.35}{B W[H z]}
$$




$$
\begin{gathered}
A(d B)_{f s}=k_{2} \cdot 20 \cdot \log _{10}\left(\frac{\sqrt{\left(C_{d} \cdot R_{d} \cdot R \cdot \omega_{f s}\right)^{2}+(R)^{2}}}{\sqrt{\left(\left(C_{d} \cdot R_{d} \cdot R+L\right) \cdot \omega_{f s}-C \cdot C_{d} \cdot R_{d} L \cdot R \cdot \omega_{f s}^{3}\right)^{2}+\left(R-\left(C \cdot L \cdot R+C_{d} \cdot L \cdot R+C_{d} \cdot L \cdot R_{d}\right) \cdot \omega_{f s}^{2}\right)^{2}}}\right) \\
k_{2}=\frac{\text { Measured } A(d B)_{f s} \text { in numerical simulation }}{\text { Predicted } A(d B)_{f s} \text { by analytical design models }}
\end{gathered}
$$

During the rise-time the losses in the damping resistance are evaluated considering the average current necessary to charge $C_{d}$ capacitor to $V_{\text {OUT }}(13)$. During the pulse, only the ripple current passes through the $C_{d}-R_{d}$ branch, so the losses during this period can be computed as shown in (14).

$$
\begin{aligned}
& P_{R_{d_{-}} \text {rise }}(s)=R_{d} \cdot\left(\frac{C_{d} \cdot V_{\text {OUT }}}{t_{\text {rise }}}\right)^{2} \cdot t_{\text {rise }} \cdot R R \\
& P_{R_{d \_} \text {pulse }}(s)=R_{d} \cdot\left(\frac{{ }^{V} \text { oUT } \frac{V_{\text {OUT }}}{V_{D C \_} B U S}}{2 . f s . L}\right)^{2} \cdot t_{\text {pulse }} \cdot R R
\end{aligned}
$$

The losses in the inductance are calculated with the assumption that there are the same losses in the magnetic core as in the copper. The required copper surface is computed supposing a current density of $2 \mathrm{~A} / \mathrm{mm}^{2}$ and a saturation flux density of $1 \mathrm{~T}$.

The conduction and switching losses in the IGBT, and the conduction and reverse recovery losses in the diode, are computed following the classical expressions presented in [10]. The selected 1200V 200A IGBT and diode for this example are the SKM200GB123D and SKM200GAL125D from Semikron, respectively. All the necessary data to compute the losses in semiconductors are taken from manufacturer's datasheets.

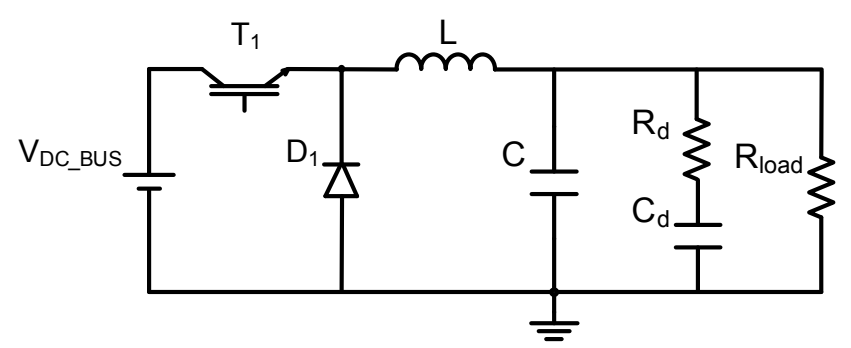

Fig. 5: Buck converter equipped with damped output filter.

Figure 6 illustrates the evolution of the overshoot peak prediction on the design models over a total of 10 corrective iteration steps. Figure 7 shows the evolution of the absolute error and the correction factor over the design method process. We observe that, after 5 corrective iteration steps, the error drops below $1 \%$, whereas the correction factor $k_{l}$ of the same figure ends at $\mathrm{k}_{1}=0.62$. 


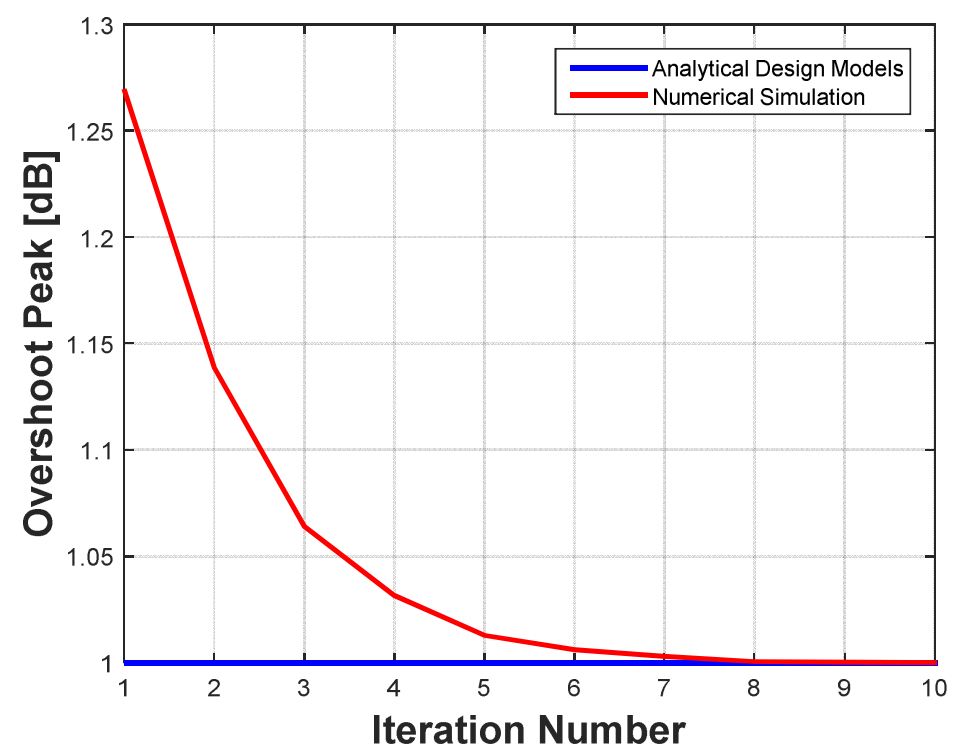

Fig. 6: Evolution of the overshoot predicted by the analytical models and the one obtained by numerical simulation.
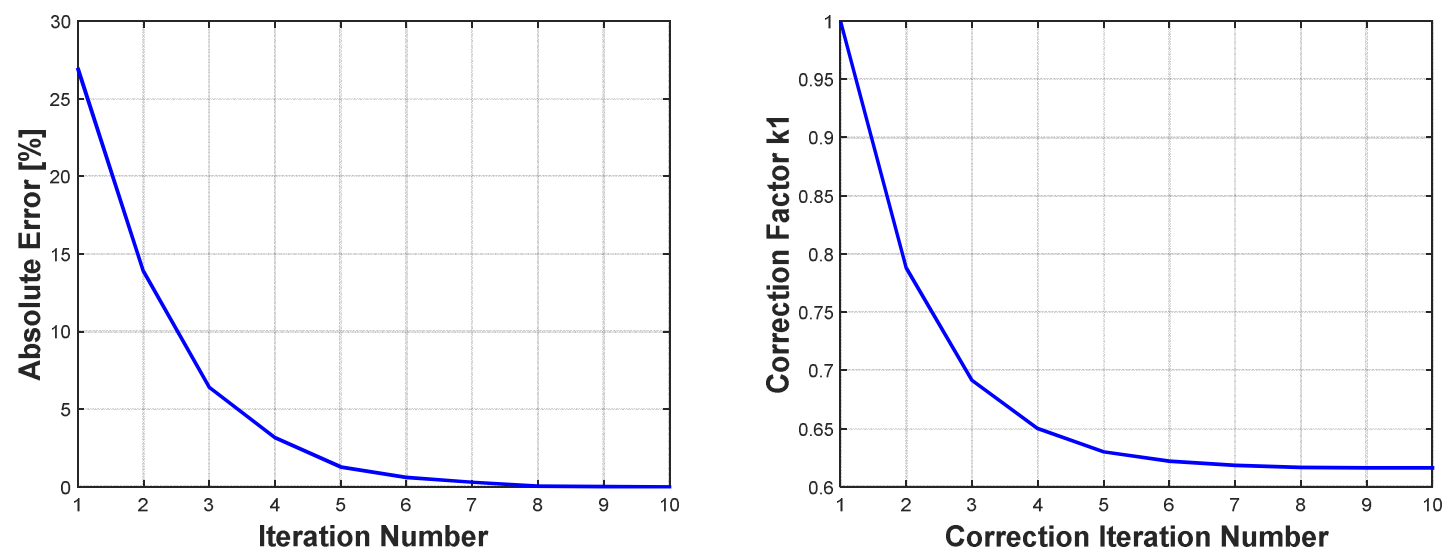

Fig. 7: Evolution of the absolute error on overshoot and correction factor during 10 iteration steps

Figure 8 shows the differences between the expected and the measured ripple attenuation over the previous 10 corrective iteration steps. The error rapidly drops to almost zero and the correction factor $k_{2}$ approaches 1, as illustrated in Figures 8 and 9. The fact that $k_{2}$ almost reaches 1 at the end of the procedure, shows that the analytical formulation for the ripple prediction is quite accurate. 


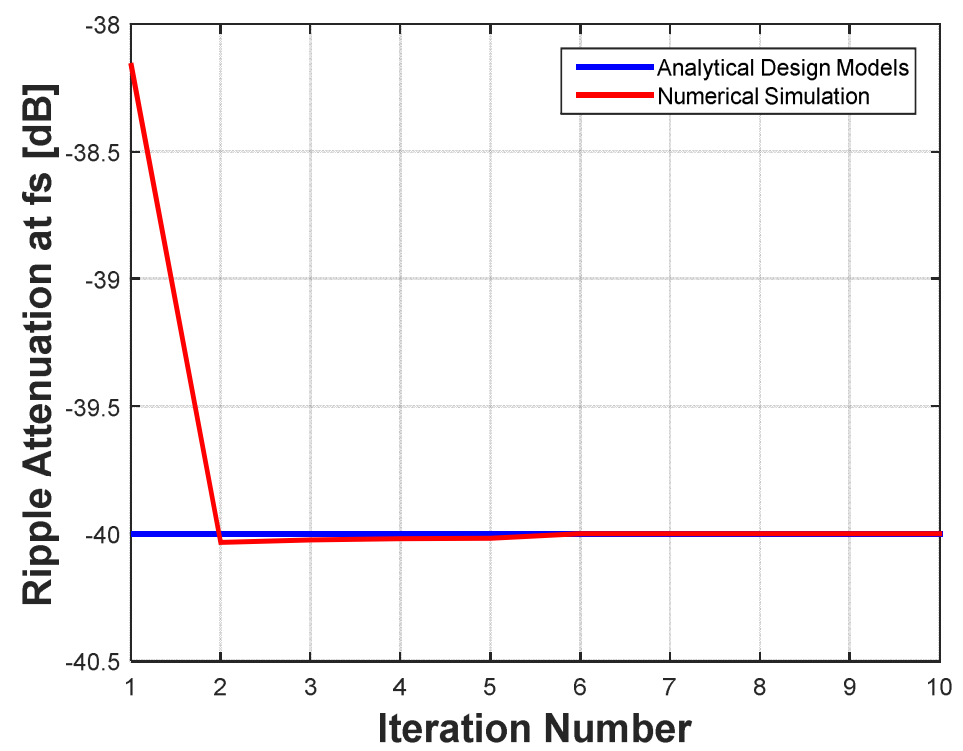

Fig. 8: Evolution of the predicted switching frequency ripple attenuation by the analytical models versus the ones obtained by numerical simulation
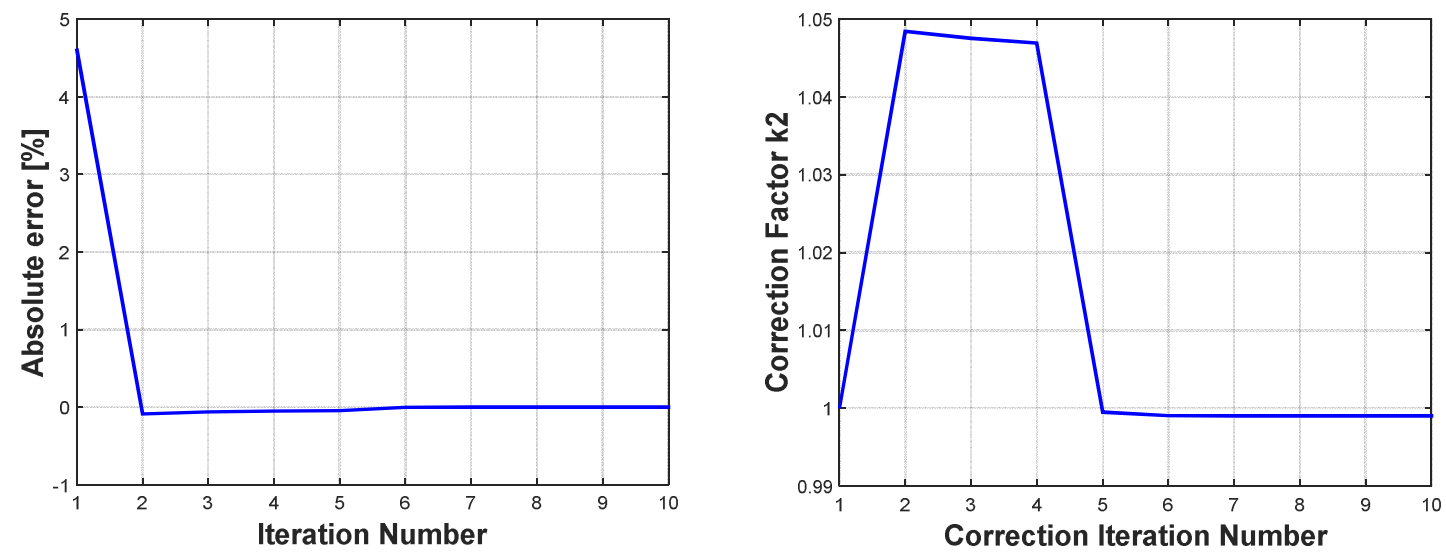

Fig. 9: Evolution of the absolute error on predicted switching frequency harmonics attenuation and the correction factor during 10 iteration steps.

\section{Conclusion}

A novel hybrid optimization method for designing power electronics converters including the controller parameters has been presented. This method combines the speed of purely analytical designmodels based optimization methods, with the precision of the time-domain numerical simulation, by means of correction factors after each intermediate optimization result. Moreover this method allows considering in a simple way, delays and other nonlinearities present in the real system, which are difficult to consider in an analytical model.

The evolution and final values of the correction factors give hints regarding the accuracy of the analytical models. It is interesting to note that in this paper the corrective actions have been carried out via multiplications with a constant value; however one can also consider a corrective action via dedicated functions, which can affect the analytical model sensitivities and have an impact the convergence of the presented hybrid optimization design process. 


\section{References}

[1] Gezgin C., Heck Bonnie S. and Bass Richard M.: Simultaneous Design of Power Stage and Controller for Switching Power Supplies, IEEE Transactions on Power Electronics, Vol. 12, No. 3, May 1997

[2] Duro B., Ramsden V.S. and Muttik P.: Minimization of Active Filter Rating in High Power Hybrid Filter Systems, IEEE International Conference on Power Electronics and Drive Systems, July 1999, Hong Kong.

[3] Busquets-Monge S., Soremekun G., Hertz E., Crebier C., Ragon S., Zhang J., Boroyevich D., Gurdal Z., Lindner D.K. and Arpilliere M.: Design optimization of a boost power factor correction converter using genetic algorithms, IEEE Applied Power Electronics Conference and Exposition, March 2002.

[4] Busquets-Monge S., Soremekun G., Hefiz E., Crebier C., Ragon S., Boroyevich D., Gurdal Z., Arpilliere M. and Lindner D. K.: Power Converter Design Optimization, IEEE Industry Applications Magazine, JanuaryFebruary 2004.

[5] Blachandran S. and Lee F. C.: Algorithms for Power Converter Design Optimization, IEEE Transactions on Aerospace and Electronic Systems, Vol. AES-17 No. 3, July 1981.

[6] Wu C.J., Lee F. C., Balachandran S. and Goin H.L.: Design Optimization for a Half-Bridge DC-DC Converter, IEEE Transaction son Aerospace and Electronic Systems, Vol. AES-18 No. 4, July 1982.

[7] Ridley R. B. and Lee F. C.: Application of Nonlinear Design Optimization for Power converter Components, IEEE Transactions on Power electronics, Vol. 5, No. 1, January 1990.

[8] Alariqi F. and Abdulrahman A.: Optimum Tuning of the PID Controller for Stable and Unstable Systems Using Nonlinear Optimization Technique, Proceedings of International MultiConference of Engineers and Computer Scientists 2014 Vol1, March 2014, Hong Kong.

[9] Radaorozandry L. E.: Conception et optimisation des machines synchrones avec des modèles analytiques en tenant compte de la saturation magnétique, M.Sc. Dissertation, Laval University, 2007, 156 p.

[10] Backlund B., Schnell R., Schlapbach U., Fisher, R. and Tsyplakov E: Applying IGBTS, Application Note, ABB Switzerland Ltd, May 2012 